\section{Should Antipsychotic Polypharmacy Be Used for Patients with Schizophrenia and Related Psychoses?}

\section{THE "PRO" SIDE}

Schizophrenia is a serious psychiatric condition characterized by psychotic symptoms (e.g., hallucinations, delusions, and disorganization), negative symptoms (e.g., anhedonia, avolition), cognitive symptoms, and deficits of executive function. ${ }^{1}$ Worldwide, schizophrenia is one of the top 20 causes of disability, partly owing to its early onset and persistent symptoms. ${ }^{2}$

Antipsychotics form the cornerstone of therapy for schizophrenia. Medications in this class are considered to have comparable effectiveness, with the exception of clozapine, ${ }^{2}$ which is the only antipsychotic agent indicated for treatment-refractory schizophrenia. ${ }^{3}$ However, the use of clozapine is complicated by significant, multifaceted barriers, including the need for frequent blood monitoring, inadequate clinician knowledge or experience, inadequate staff resources to support the use of clozapine therapy, and service fragmentation. ${ }^{4}$ Such barriers limit the feasibility of clozapine therapy for many patients. As a result, clinicians may administer other antipsychotics in combination to relieve patients' distressing symptoms.

Antipsychotic polypharmacy (APP) is defined as the concurrent use of 2 or more antipsychotics. The prevalence of APP is estimated to range from $12.9 \%$ to $35 \% .{ }^{5}$ In a 2010 Canadian study, prevalence was estimated at $25.7 \%$ in a single metropolitan area. ${ }^{6}$ Despite APP being common practice, international guidelines have provided little guidance. The 2017 Canadian schizophrenia guidelines ${ }^{7}$ and the 2021 American Psychiatric Association guideline ${ }^{2}$ provide no firm recommendations on APP; in contrast, the 2019 guidelines of the British Association for Psychopharmacology stated that APP can be used but requires careful monitoring. ${ }^{8}$ As pharmacists, we may view the prescribing of two agents within a therapeutic class as duplicate therapy. However, we should thoroughly examine the evidence for APP to better appreciate its role in the care of patients with schizophrenia.

Recent studies have attempted to evaluate the efficacy and safety of APP. Notably, the Cochrane Collaboration recently reviewed antipsychotic combinations for schizophrenia. ${ }^{9}$ Comparing antipsychotic combination therapy with monotherapy, the authors found a $27 \%$ relative reduction in the risk of having no clinical response, with a number needed to treat of 9 . The benefit was more pronounced when an antipsychotic was added to clozapine, and combinations involving clozapine had a number needed to treat of 7. Concerns were raised about the high risk of bias in the studies included in the review and inconsistencies in terms of observed benefits related to various outcomes. Consequently, firm conclusions could not be drawn to inform clinicians, despite the clinically relevant benefit. This uncertainty adds to the confusion surrounding APP, despite some evidence of benefit.

In situations where meta-analysis of randomized studies is inconclusive, well-designed observational research can fill the knowledge gap. In the usual evidence-based medicine paradigm, meta-analyses of randomized controlled trials are considered to be the top of the evidence hierarchy. However, owing to the stringent inclusion and exclusion criteria used in randomized studies, trial participants are not always representative of the patients in clinical practice. One exploratory study found that only $13.5 \%$ of all patients in a psychiatric clinic were recruited into a study performed in that clinic, which suggests significant selection bias. ${ }^{10}$ In fact, studies often exclude patients with history of nonadherence, comorbid substance use, and unstable living situations, all of which frequently co-occur for patients with schizophrenia. Such exclusions call into question whether efficacy studies reflect true effectiveness in schizophrenia research. Observational studies may better reflect clinical practice because they involve real-world patients. This is perhaps best highlighted by the evidence for long-acting depot antipsychotics: randomized data have found no evidence of benefit, but multiple observational studies have shown benefits. ${ }^{11}$ The reason for discrepant findings is likely that nonadherent patients, who may benefit from long-acting depot antipsychotics, are not enrolled in randomized studies.

APP may be another example where observational studies provide better estimates of effect. In 2019, Tiihonen and others ${ }^{12}$ published a nationwide study from Finland exploring the effectiveness of APP. This large study, involving 62250 people with schizophrenia, systematically assessed the risk of hospitalization associated with individual antipsychotics and combinations of these drugs. The study employed within-individual analyses and other rigorous methods to address confounders and selection bias. Relative to monotherapy, combination therapy was found to reduce the risk of psychiatric rehospitalization by $7 \%$ and all-cause hospitalization by $9 \% .{ }^{12}$ These results reaffirmed 
the findings of the aforementioned meta-analysis by the Cochrane Collaboration. ${ }^{9}$ Intriguingly, combining clozapine with aripiprazole was found to reduce the risk of psychiatric hospitalization more than any monotherapy. ${ }^{12}$ This nationwide observational study highlighted the real-world benefits of APP, and clinicians can thus be reassured that APP can indeed improve patient outcomes.

The evidence supporting the benefits of APP has been mounting, although valid concerns have been raised about its safety. ${ }^{13}$ Reassuringly, recent meta-analyses have found no evidence of increased serious adverse events or discontinuation of therapy due to adverse events., ${ }^{9,14}$ There was also no evidence of increased somatic hospitalization with antipsychotic combinations, relative to no antipsychotic therapy, in the Finnish study. ${ }^{12}$ Interestingly, addition of a dopamine-2 (D2) partial agonist (e.g., aripiprazole) has been found to improve prolactin levels in antipsychotic-induced hyperprolactinemia. ${ }^{14}$ Overall, the current evidence suggests that the tolerability of APP is similar to that of monotherapy.

From a psychopharmacological perspective, clinicians tend to combine antipsychotics that have different receptor affinity profiles (D2 receptors, serotonin receptors) and different side effect profiles (e.g., movement, metabolic). As a class, the antipsychotics consist of individual agents with different receptor affinities. ${ }^{2}$ The dopamine hypothesis of psychosis highlights D2 receptors as important therapeutic targets, and antipsychotic agents have variable activity on these receptors. ${ }^{1,2}$ Additionally, antipsychotics differ in their affinities for other dopamine receptor subtypes, as well as for serotonin receptor subtypes. ${ }^{2}$ While it may seem counterintuitive to combine two medications from the same therapeutic class, APP takes advantage of the different pharmacological profiles of each agent, such as combining clozapine (low D2 affinity) with risperidone (high D2 affinity). Given their expertise in pharmacology, pharmacists can play an important role in choosing antipsychotic combinations.

Although APP ought not to be the default treatment for patients with schizophrenia, it can be helpful in certain patients who experience no response to monotherapy. Growing evidence supports combining clozapine with another antipsychotic for additional benefits. ${ }^{9}, 12$ Given the suffering and unmet needs that can result from uncontrolled schizophrenia symptoms, APP can have a significant positive impact on a patient's life. It is therefore time to consider APP as a viable therapeutic option to improve patient outcomes.

\section{References}

1. Marder SR, Cannon TD. Schizophrenia. N Engl J Med. 2019;381(18): 1753-61.

2. Practice guideline for the treatment of patients with schizophrenia. $3 \mathrm{rd}$ ed. American Psychiatric Association; @ 2021 [cited 2020 Nov 6]. Available from: https://psychiatryonline.org/doi/book/10.1176/appi.books .9780890424841

3. Kane J, Honigfeld G, Singer J, Meltzer H. Clozapine for the treatmentresistant schizophrenic. A double-blind comparison with chlorpromazine. Arch Gen Psychiatry. 1988;45(9):789-96.
4. Farooq S, Choudry A, Cohen D, Naeem F, Ayub M. Barriers to using clozapine in treatment-resistant schizophrenia: systematic review. BJPsych Bull. 2019;43(1):8-16.

5. Gallego JA, Bonetti J, Zhang J, Kane JM, Correll CU. Prevalence and correlates of antipsychotic polypharmacy: a systematic review and meta-regression of global and regional trends from the 1970s to 2009 . Schizophr Res. 2012;138(1):18-28.

6. Procyshyn RM, Honer WG, Wu TKY, Ko RWY, McIsaac SA, Young AH, et al. Persistent antipsychotic polypharmacy and excessive dosing in the community psychiatric treatment setting: a review of medication profiles in 435 Canadian outpatients. J Clin Psychiatry. 2010;71(5):566-73.

7. Remington G, Addington D, Honer W, Ismail Z, Raedler T, Teehan M. Guidelines for the pharmacotherapy of schizophrenia in adults. Can J Psychiatry. 2017;62(9):604-16.

8. Barnes TR, Drake R, Paton C, Cooper SJ, Deakin B, Ferrier IN, et al. Evidence-based guidelines for the pharmacological treatment of schizophrenia: updated recommendations from the British Association for Psychopharmacology. J Psychopharmacol. 2020;34(1):3-78.

9. Ortiz-Orendain J, Castiello-de Obeso S, Colunga-Lozano LE, Hu Y, Maayan N, Adams CE. Antipsychotic combinations for schizophrenia. Cochrane Database Sys Rev. 2017;6(6):CD009005.

10. Hofer A, Hummer M, Huber R, Kurz M, Walch T, Fleischhacker W. Selection bias in clinical trials with antipsychotics. JClin Psychopharmacol. 2000;20(6):699-702.

11. Kane JM, Kishimoto T, Correll CU. Assessing the comparative effectiveness of long-acting injectable vs. oral antipsychotic medications in the prevention of relapse provides a case study in comparative effectiveness research in psychiatry. J Clin Epidemiol. 2013;66(8 Suppl):S37-41.

12. Tiihonen J, Taipale H, Mehtälä J, Vattulainen P, Correll CU, Tanskanen A. Association of antipsychotic polypharmacy vs monotherapy with psychiatric rehospitalization among adults with schizophrenia. JAMA Psychiatry. 2019;76(5):499-507.

13. Gallego JA, Nielsen J, De Hert M, Kane JM, Correll CU. Safety and tolerability of antipsychotic polypharmacy. Expert Opin Drug Saf. 2012; 11(4):527-42.

14. Galling B, Roldán A, Rietschel L, Hagi K, Walyzada F, Zheng W, et al. Safety and tolerability of antipsychotic co-treatment in patients with schizophrenia: results from a systematic review and meta-analysis of randomized controlled trials. Expert Opin Drug Saf. 2016;15(5):591-612.

Jacky T P Siu, BScPharm, ACPR, PharmD, BCPP

Clinical Pharmacy Specialist, Tertiary Mental Health and Substance Use Lower Mainland Pharmacy Services

Clinical Assistant Professor, Faculty of Pharmaceutical Sciences

The University of British Columbia

Vancouver, British Columbia

Competing interests: None declared.

\section{THE "CON" SIDE}

Antipsychotic polypharmacy (APP) is a common practice in treating schizophrenia. In particular, treatment-resistant schizophrenia accounts for about $30 \%$ of all patients with schizophrenia, and these patients often receive antipsychotic combination therapy, even though clozapine (the first-line therapy) is the only medication approved for this indication. However, before starting clozapine, about $60 \%$ of patients receive treatment with combinations of antipsychotics, an approach that is not based on guideline recommendations. ${ }^{1}$ Clozapine is widely underutilized, with many clinicians opting to increase current antipsychotic doses, add a second antipsychotic, change to a long-acting injection formulation, 
or add a mood stabilizer before starting a clozapine trial. ${ }^{2}$ Up to 4 non-clozapine antipsychotics may be trialled before clozapine initiation, which can lead to delays in appropriate treatment of 2 to 5 years after diagnosis of treatment-resistant schizophrenia. ${ }^{3}$ Delays in initiating clozapine are associated with poor response, and patients gain less benefit from clozapine if there is a delay. ${ }^{1}$ Yoshimura and others ${ }^{4}$ found an $81.6 \%$ response rate when clozapine was started within a window of 2.8 years, compared with response rates of only $30.8 \%$ among those with clozapine initiation delayed beyond 2.8 years. Barriers to clozapine initiation include prescribers' fears, local practices, geographic location, and lack of resources supporting the services required by patients receiving clozapine. ${ }^{5}$ Given that clozapine is the only therapy with proven benefit in this population, it is imperative that these barriers be addressed to improve clinical outcomes and minimize the delay in effective therapy.

Current antipsychotic therapies primarily improve the positive symptoms of schizophrenia (e.g., hallucinations, delusions). A 2017 Cochrane meta-analysis on the use of APP showed benefits in terms of symptom improvement, but the evidence came from low-quality, open-label studies that included heterogeneous patient populations with various phases of illness. ${ }^{6}$ Additionally, clozapine and non-clozapine combinations were used, thus making it difficult to apply the findings to treatment-resistant or treatment-responsive patients. ${ }^{6}$ There were also few data related to functional outcomes. Functional status is a critical domain of schizophrenia that is not well characterized and is seldom included as a study outcome. In the few reviews of APP that have assessed functional outcomes (e.g., Global Assessment of Functioning scale), there was no difference between APP and monotherapy, likely because the studies included in the reviews were small and short in duration. ${ }^{6}$ Even well-designed observational data should be interpreted with caution. Tiihonen and others ${ }^{7}$ found that APP significantly reduced the risk of psychiatric hospitalizations, with clozapine and aripiprazole being the best combination for this result. However, a closer look at the data shows that there may be no difference between clozapine monotherapy and clozapine combinations, given the overlap of confidence intervals (CIs). Furthermore, the minimal benefit of combining clozapine and aripiprazole (hazard ratio [HR] 0.42, 95\% CI 0.39-0.46) relative to clozapine monotherapy (HR 0.49 , 95\% CI 0.47 $0.51)$ may have been due to residual confounding. ${ }^{7}$

APP is a major contributor to high-dose prescribing, which often exceeds the maximum licensed daily dose and defined daily dose. ${ }^{8}$ The defined daily dose is the "assumed average maintenance dose per day for a drug used for its main indication in adults" ${ }^{\prime \prime}$ and is used to compare the cumulative dose of antipsychotic therapy, either as a single agent or as part of a polypharmacy regimen. ${ }^{10}$ Antipsychotic dosing is considered excessive when the ratio of prescribed daily dose to defined daily dose (PDD/DDD) is greater than 1.5. ${ }^{10}$ Procyshyn and others ${ }^{10}$ found that patients with APP received persistently high doses of antipsychotics, with a $\mathrm{PDD} / \mathrm{DDD}$ ratio of 1.94 , whereas PDD/DDD was 0.94 for those receiving antipsychotic monotherapy. Specifically, higher proportions of patients with schizophrenia would receive excessive daily dosing compared with other indications for antipsychotics (e.g., bipolar disorder). ${ }^{10} \mathrm{~A}$ doseresponse meta-analysis of antipsychotic drugs showed that doses identified to be $95 \%$ effective were often lower than the recommended maximum doses, whereas doses higher than an equivalent of 3-5 $\mathrm{mg}$ of risperidone provide limited additional benefit ${ }^{11}$ and led to significant concerns for increased adverse effects.

APP is associated with increased movement, metabolic, cardiac, and neurocognitive adverse effects, ${ }^{12}$ which can lead to additional medication therapy. In particular, anticholinergic medications are often prescribed to alleviate extrapyramidal symptoms caused by antipsychotics. With higher rates of extrapyramidal symptoms, the use of anticholinergic agents increases, often also at high doses. ${ }^{12}$ Antipsychotics themselves have anticholinergic activity at the muscarinic receptors, which adds to the burden of anticholinergic-related adverse effects such as cognitive impairment, constipation, dry mouth, urinary retention, and blurred vision. The burden of adverse effects is one of the most commonly reported reasons for medication non-adherence, with dry mouth and sexual dysfunction being significantly more common in patients receiving polypharmacy. ${ }^{13}$

Reducing high doses of antipsychotics and switching to monotherapy can be accomplished safely. Essock and others ${ }^{14}$ showed that patients who were switched from polypharmacy to monotherapy had shorter time to all-cause discontinuation, with about $20 \%$ in the "switch to monotherapy" group reverting to their original polytherapy regimen. However, this was an open-label study in which patients and assessors were biased in terms of associating changes in symptoms with changes in medications. In fact, of patients assigned to the "switch to monotherapy" group, $69 \%$ were able to successfully switch to monotherapy with no change in symptoms or psychiatric hospitalizations relative to the "stay on polypharmacy" group. ${ }^{14}$ Additionally, Yamanouchi and others ${ }^{15}$ demonstrated that alternative methods, such as gradual dose reduction over 12 weeks, can also be successful in reducing high doses related to polypharmacy, with no worsening of psychotic symptoms.

Overall, there is currently limited evidence to support the use of APP in most patients with schizophrenia. Although there is considerable evidence related to the use of APP, it is flawed and difficult to apply, and it does not include many clinically important outcomes (e.g., functional outcomes). In addition to significant safety concerns, APP delays initiation of effective monotherapies (e.g., clozapine), and efforts should be made to optimize trials of monotherapy before adding more antipsychotics to a patient's regimen. 


\section{References}

1. Üçok A, Çikrikçili U, Karabulut S, Salaj A, Öztürk M, Tabak Ö, et al. Delayed initiation of clozapine may be related to poor response in treatment-resistant schizophrenia. Int Clin Psychopharmacol. 2015; 30(5):290-5.

2. Correll CU, Brevig T, Brain C. Patient characteristics, burden and pharmacotherapy of treatment-resistant schizophrenia: results from a survey of 204 US psychiatrists. BMC Psychiatry. 2019;19:Article 362.

3. Shah P, Iwata Y, Plitman E, Brown EE, Caravaggio F, Kim J, et al. The impact of delay in clozapine initiation on treatment outcomes in patients with treatment-resistant schizophrenia: a systematic review. Psychiatry Res. 2018;268:114-22.

4. Yoshimura B, Yada Y, So R, Takaki M, Yamada N. The critical treatment window of clozapine in treatment-resistant schizophrenia: secondary analysis of an observational study. Psychiatry Res. 2017;250:65-70.

5. Stroup TS, Gerhard T, Crystal S, Huang C, Olfson M. Geographic and clinical variation in clozapine use in the United States. Psychiatr Serv. 2014;65(2):186-92.

6. Ortiz-Orendain J, Castiello-de Obeso S, Colunga-Lozano L, Hu Y, Maayan N, Adams C. Antipsychotic combinations for schizophrenia. Schizophr Bull. 2018;44(1):15-7.

7. Tiihonen J, Taipale H, Mehtälä J, Vattulainen P, Correll CU, Tanskanen A. Association of antipsychotic polypharmacy vs monotherapy with psychiatric rehospitalization among adults with schizophrenia. JAMA Psychiatry. 2019;76(5):499-507.

8. Nguyen ML, Sunderland B, Lim S, Hattingh L, Chalmers L. The hidden magnitude of polypharmacy: using defined daily doses and maximum licensed daily doses to measure antipsychotic load. Int J Clin Pharm. 2019;41(6):1642-51.

9. Defined daily dose: definition and general considerations. WHO Collaborating Centre for Drug Statistics Methodology; [cited 2020 Nov 17]. Available from: https://www.whocc.no/ddd/definition_and_general_considera/
10. Procyshyn RM, Honer WG, Wu TKY, Ko RWY, McIsaac SA, Young AH, et al. Persistent antipsychotic polypharmacy and excessive dosing in the community psychiatric treatment setting: a review of medication profiles in 435 Canadian outpatients. J Clin Psychiatry. 2010;71(5):566-73.

11. Leucht S, Crippa A, Siafis S, Patel MX, Orsini N, Davis JM. Doseresponse meta-analysis of antipsychotic drugs for acute schizophrenia. Am J Psychiatry. 2020;177(4):342-53.

12. Gallego JA, Nielsen J, De Hert M, Kane JM, Correll CU. Safety and tolerability of antipsychotic polypharmacy. Expert Opin Drug Saf. 2012; 11(4):527-42.

13. Hashimoto Y, Uno J, Miwa T, Kurihara M, Tanifuji H, Tensho M. Effects of antipsychotic polypharmacy on side-effects and concurrent use of medications in schizophrenic outpatients. Psychiatry Clin Neurosci. 2012;66(5):405-10.

14. Essock SM, Schooler NR, Stroup TS, McEvoy JP, Rojas I, Jackson C, et al. Effectiveness of switching from antipsychotic polypharmacy to monotherapy. Am J Psychiatry. 2011;168(7):702-8.

15. Yamanouchi $Y$, Sukegawa T, Inagaki A, Inada T, Yoshio T, Yoshimura $\mathrm{R}$, et al. Evaluation of the individual safe correction of antipsychotic agent polypharmacy in Japanese patients with chronic schizophrenia: validation of safe corrections for antipsychotic polypharmacy and the high-dose method. Int J Neuropsychopharmacol. 2015;18(5):pyu016.

Anna Yee, BSc(Pharm), ACPR, PharmD Fraser Health

Lower Mainland Pharmacy Services

Vancouver, British Columbia

Competing interests: None declared.

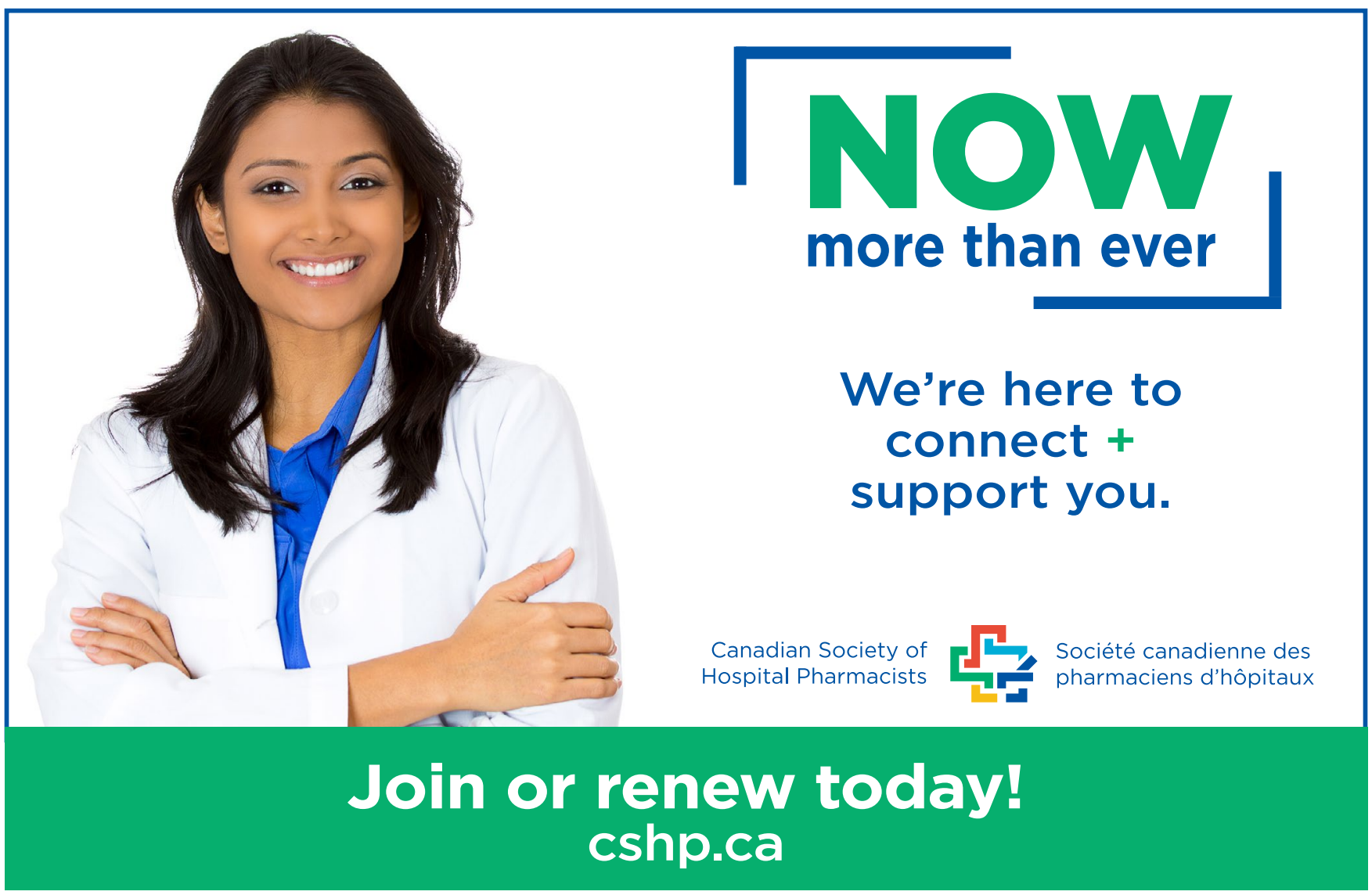

